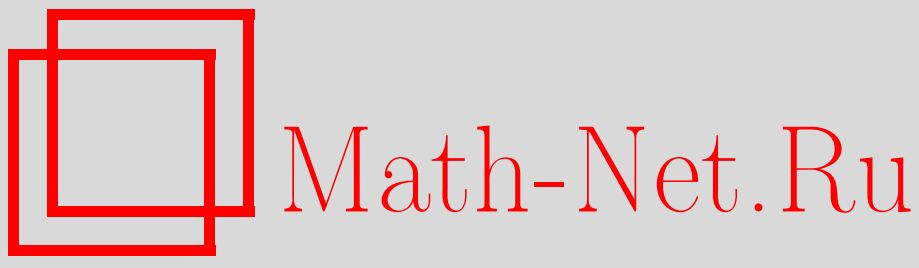

А. А. Ковалевский, О суммируемости энтропийных решений задачи Дирихле для одного класса нелинейных эллиптических уравнений четвертого порядка, Изв. РАН. Сер. матем., 2003, том 67, выпуск 5, 35-48

DOI: https://doi.org/10.4213/im450

Использование Общероссийского математического портала Math-Net.Ru подразумевает, что вы прочитали и согласны с пользовательским соглашением http://www . mathnet.ru/rus/agreement

Параметры загрузки:

IP: 107.22 .136 .117

26 апреля 2023 г., 13:17:06 
УДК 517.9

\title{
А. А. Ковалевский \\ О суммируемости энтропийных решений задачи Дирихле для одного класса нелинейных эллиптических уравнений четвертого порядка
}

\begin{abstract}
Приведены результаты о суммируемости энтропийных решений задачи Дирихле для некоторого класса нелинейных эллиптических уравнений четвертого порядка в зависимости от свойств интегрируемости правых частей уравнений. Рассмотрены случаи, когда эти правые части принадлежат пространствам Лебега с показателями, близкими к единице, а также более широким множествам, содержащимся в $L^{1}$.

Библиографиия: 8 наименований.
\end{abstract}

\section{Введение}

Понятие энтропийного решения задачи Дирихле для рассматриваемого в настоящей статье класса нелинейных эллиптических уравнений четвертого порядка введено и изучено в [1], [2] на основе подхода, предложенного в [3] для уравнений второго порядка. В работе получен ряд результатов о суммируемости энтропийных решений данной задачи в зависимости от свойств интегрируемости правых частей соответствуюших уравнений. Рассмотрены случаи, когда эти правые части принадлежат пространствам Лебега с показателями, близкими к единице, а также более широким множествам, содержашимся в $L^{1}$. Отметим, что для нелинейных эллиптических уравнений второго порядка с правыми частями такого типа некоторые результаты о суммируемости слабых решений получены в [4], [5]. Недавно они были уточнены и дополнены в работе [6] на основе полученных в ней результатов о суммируемости энтропийных решений тех же уравнений.

Настоящая статья состоит из двух параграфов. В $\S 1$ рассмотрено множество функций $\stackrel{\circ}{H}_{2, p}^{1, q}(\Omega)$, введенное в [1], [2], и для элементов этого множества, подчиненных некоторым интегральным неравенствам, установлены различные свойства интегрируемости. При этом используются приемы, аналогичные изложенным в [6] для иной ситуации. В $\S 2$ приведена формулировка рассматриваемой задачи Дирихле и установлено, что энтропийные решения этой задачи удовлетворяют интегральным неравенствам из $\S 1$. Этот факт вместе с надлежащими условиями на правые части рассматриваемых уравнений и результатами, полученными в 1 , позволяет доказать теоремы об интегрируемости энтропийных решений данной задачи. Приведены также некоторые результаты для слабых решений этой задачи.

Исходные предположения работы следующие: $n \in \mathbb{N}, n>2, \Omega$-ограниченное открытое множество в $\mathbb{R}^{n}, p \in(1, n / 2), q \in(2 p, n)$.

(C) А.А. КовАЛЕвСКий, 2003 
Пусть $\Lambda$ - множество всех $n$-мерных мультииндексов $\alpha$ таких, что $|\alpha|=1$ или $|\alpha|=2$, а $\mathbb{R}^{n, 2}$ - пространство всех отображений $\xi: \Lambda \rightarrow \mathbb{R}$. Если $u \in W^{2,1}(\Omega)$, то $\nabla_{2} u: \Omega \rightarrow \mathbb{R}^{n, 2}$, причем для любых $x \in \Omega$ и $\alpha \in \Lambda$ имеем $\left(\nabla_{2} u(x)\right)_{\alpha}=D^{\alpha} u(x)$.

\section{$\S 1$. О суммируемости функций, подчиненных некоторым интегральным неравенствам}

Приведем сначала несколько общих результатов, которые будут полезны в дальнейшем.

ЛЕмма 1.1. Пусть и-измеримая функиия на $\Omega, \quad M>0, \tau>0$, и пусть для любого $k \in \mathbb{N}$ справедливо неравенство

$$
\text { meas }\{|u| \geqslant k\} \leqslant M k^{-\tau} \text {. }
$$

Тогда для любого $\lambda \in(0, \tau)$ имеем $u \in L^{\lambda}(\Omega)$.

ДокаЗАТЕЛЬСтво. Зафиксируем $\lambda \in(0, \tau)$ и положим $\lambda_{1}=2 /(\tau-\lambda)$. В силу (1.1) для любого $k \in \mathbb{N}$ имеем

$$
\int_{\left\{k^{\lambda_{1}} \leqslant|u|<(k+1)^{\left.\lambda_{1}\right\}}\right.}|u|^{\lambda} d x \leqslant 2^{\tau+\lambda_{1} \lambda} M k^{-2} .
$$

Отсюда, производя суммирование по $k$ в обеих частях неравенства, заключаем, что $u \in L^{\lambda}(\Omega)$. Лемма доказана.

Отметим, что свойство, представленное леммой 1.1 , использовалось ранее, например в [3], а также в [1], [2].

Лемма 1.2. Пусть $и$-измеримая функция на $\Omega, \quad M>0, \tau>0, \tau_{1}>1, u$ пусть для любого $k \in \mathbb{N}, k \geqslant 2$, справедливо неравенство

$$
\text { meas }\{|u| \geqslant k\} \leqslant M k^{-\tau}(\ln k)^{-\tau_{1}} \text {. }
$$

Тогда $u \in L^{\tau}(\Omega)$.

ДокаЗАТЕЛЬСтво. В силу (1.2) для любого $k \in \mathbb{N}$ имеем

$$
\int_{\left\{e^{k} \leqslant|u|<e^{k+1}\right\}}|u|^{\tau} d x \leqslant 2^{\tau+\tau_{1}} e^{\tau} M k^{-\tau_{1}} .
$$

Отсюда, производя суммирование по $k$ в обеих частях неравенства и учитывая, что $\tau_{1}>1$, получаем, что $u \in L^{\tau}(\Omega)$. Лемма доказана.

Для любого $\lambda \in[1, n)$ положим $\lambda^{*}=n \lambda /(n-\lambda)$.

Известно (см., например, [7]), что если $\lambda \in[1, n)$, то $\stackrel{\circ}{W^{1, \lambda}}(\Omega) \subset L^{\lambda^{*}}(\Omega)$ и существует положительная постоянная $c_{n, \lambda}$, зависяшая только от $n$ и $\lambda$, такая, что для любой функции $u \in \stackrel{\circ}{W^{1, \lambda}}(\Omega)$

$$
\left(\int_{\Omega}|u|^{\lambda^{*}} d x\right)^{1 / \lambda^{*}} \leqslant c_{n, \lambda}\left(\sum_{|\alpha|=1} \int_{\Omega}\left|D^{\alpha} u\right|^{\lambda} d x\right)^{1 / \lambda} .
$$


Перейдем к рассмотрению некоторого специального множества функций и свойств его элементов.

Через $W_{2, p}^{1, q}(\Omega)$ обозначим множество всех функций из $W^{1, q}(\Omega)$, имеющих обобщенные производные второго порядка из $L^{p}(\Omega)$. Множество $W_{2, p}^{1, q}(\Omega)$ - банахово пространство с нормой

$$
\|u\|=\|u\|_{W^{1, q}(\Omega)}+\left(\sum_{|\alpha|=2} \int_{\Omega}\left|D^{\alpha} u\right|^{p} d x\right)^{1 / p} .
$$

Через $\stackrel{\circ}{W} \underset{2, p}{1, q}(\Omega)$ обозначим замыкание в $W_{2, p}^{1, q}(\Omega)$ множества $C_{0}^{\infty}(\Omega)$.

Пусть для любого $k \in \mathbb{N} \psi_{k}$ - функция на $\mathbb{R}$ такая, что

$$
\psi_{k}(s)=s-s^{k+2}+\frac{k+1}{k+3} s^{k+3}, \quad s \in \mathbb{R} .
$$

Определим теперь для любого $k \in \mathbb{N}$ функцию $h_{k}: \mathbb{R} \rightarrow \mathbb{R}$, полагая

$$
h_{k}(s)= \begin{cases}s, & \text { если }|s| \leqslant k, \\ {\left[\psi_{k}\left(\frac{|s|-k}{k}\right)+1\right] k \operatorname{sign} s,} & \text { если } k<|s|<2 k, \\ 2 k \frac{k+2}{k+3} \operatorname{sign} s, & \text { если }|s| \geqslant 2 k .\end{cases}
$$

Для любого $k \in \mathbb{N}$ имеем $h_{k} \in C^{2}(\mathbb{R})$ и, кроме того,

$$
\begin{array}{rll}
\left|h_{k}\right| \leqslant 2 k & \text { на } & \mathbb{R} \\
0 \leqslant h_{k}^{\prime} \leqslant 1 & \text { на } & \mathbb{R} \\
\left|h_{k}^{\prime \prime}\right| \leqslant 3 & \text { на } & \mathbb{R} .
\end{array}
$$

Через $\stackrel{\circ}{H}{ }_{2, p}^{1, q}(\Omega)$ обозначим множество всех функций $u: \Omega \rightarrow \mathbb{R}$, удовлетворяющих следующему условию: $h_{k}(u) \in \stackrel{\circ}{W_{2, p}^{1, q}}(\Omega) \forall k \in \mathbb{N}$.

Положим для произвольных $u: \Omega \rightarrow \mathbb{R}$ и $x \in \Omega$

$$
k(u, x)=\min \{N \in \mathbb{N}:|u(x)|<N\} .
$$

ОПРедЕЛЕниЕ 1.1. Пусть $u \in \stackrel{\circ}{H} \underset{2, p}{1, q}(\Omega)$ и $\alpha \in \Lambda$. Тогда $\delta^{\alpha} u-$ функция на $\Omega$ такая, что для любого $x \in \Omega$

$$
\delta^{\alpha} u(x)=D^{\alpha} h_{k(u, x)}(u)(x) .
$$

Лемма 1.3. Пусть $u \in \stackrel{\circ}{H_{2}^{1, q}}(\Omega)$ u $\alpha \in \Lambda$. Тогда для любого $k \in \mathbb{N}$ имеем

$$
\delta^{\alpha} u=D^{\alpha} h_{k}(u) \quad \text { n.в. на }\{|u| \leqslant k\} .
$$


ДокаЗАТЕЛЬСтво. Для любых $k, k_{1} \in \mathbb{N}$ таких, что $2 k \leqslant k_{1}$, имеем $h_{k}(u)=$ $h_{k}\left(h_{k_{1}}(u)\right)$. Используя этот факт, устанавливаем, что для любых $k, k_{1} \in \mathbb{N}$ таких, что $k<k_{1}$,

$$
D^{\alpha} h_{k}(u)=D^{\alpha} h_{k_{1}}(u) \text { п.в. на }\{|u| \leqslant k\} .
$$

Зафиксируем $k \in \mathbb{N}$. В силу (1.8) сушествует множество $E$ меры нуль такое, что для любых $N \in \mathbb{N}, N<k+1$, и $x \in\{|u| \leqslant N\} \backslash E$

$$
D^{\alpha} h_{N}(u)(x)=D^{\alpha} h_{k+1}(u)(x) .
$$

Тогда для любого фиксированного $x \in\{|u| \leqslant k\} \backslash E$, учитывая определение функции $\delta^{\alpha} u$, получаем $\delta^{\alpha} u(x)=D^{\alpha} h_{k+1}(u)(x)$. Ясно также, что $D^{\alpha} h_{k}(u)(x)=$ $D^{\alpha} h_{k+1}(u)(x)$. Следовательно, $\delta^{\alpha} u(x)=D^{\alpha} h_{k}(u)(x)$. Отсюда заключаем, что (1.7) справедливо. Лемма доказана.

Отметим, что в силу леммы 1.3 приведенное выше определение функций $\delta^{\alpha} u$ эквивалентно определению этих функций, данному в [1], [2].

Лемма 1.4. Пусть $u \in \stackrel{\circ}{H_{2}^{1, q}}(\Omega), \quad k \in \mathbb{N}$. Тогда:

1) если $\alpha$-n-мерный мультииндекс, $|\alpha|=1$, то

$$
D^{\alpha} h_{k}(u)=h_{k}^{\prime}(u) \delta^{\alpha} u \quad \text { n.в. на } \Omega
$$

2) если $\alpha$-n-мерный мультииндекс, $|\alpha|=2$, то

$$
\left|D^{\alpha} h_{k}(u)\right| \leqslant\left|\delta^{\alpha} u\right|+3 \sum_{|\beta|=1}\left|\delta^{\beta} u\right|^{2} \quad \text { п.в. на } \Omega .
$$

ДокАЗАТЕЛЬСТвО. Имеем

$$
h_{k}(u)=h_{k}\left(h_{2 k}(u)\right) .
$$

Пусть $\alpha-n$-мерный мультииндекс, $|\alpha|=1$. В силу (1.9)

$$
D^{\alpha} h_{k}(u)=h_{k}^{\prime}\left(h_{2 k}(u)\right) D^{\alpha} h_{2 k}(u) \text { п.в. на } \Omega .
$$

Отсюда, используя свойства функций $h_{k}, h_{2 k}$ и лемму 1.3, получаем, что $D^{\alpha} h_{k}(u)$ $=h_{k}^{\prime}(u) \delta^{\alpha} u$ п.в. на $\Omega$, и тем самым утверждение 1$)$ доказано.

Пусть теперь $\alpha-n$-мерный мультииндекс, $|\alpha|=2$. Используя (1.9), получаем

$$
\left|D^{\alpha} h_{k}(u)-h_{k}^{\prime}\left(h_{2 k}(u)\right) D^{\alpha} h_{2 k}(u)\right| \leqslant\left|h_{k}^{\prime \prime}\left(h_{2 k}(u)\right)\right| \sum_{|\beta|=1}\left|D^{\beta} h_{2 k}(u)\right|^{2} \text { п.в. на } \Omega \text {. }
$$

Отсюда, учитывая (1.5), (1.6) и лемму 1.3 , выводим, что $\left|D^{\alpha} h_{k}(u)\right| \leqslant\left|\delta^{\alpha} u\right|+$ $3 \sum_{|\beta|=1}\left|\delta^{\beta} u\right|^{2}$ п.в. на $\Omega$, и тем самым утверждение 2$)$ доказано.

Отметим, что

$$
\stackrel{\circ}{W}_{2, p}^{1, q}(\Omega) \subset \stackrel{\circ}{H}_{2, p}^{1, q}(\Omega)
$$

и если $u \in \stackrel{\circ}{W} \underset{2, p}{1, q}(\Omega), \alpha \in \Lambda$, то $\delta^{\alpha} u=D^{\alpha} u$ п.в. на $\Omega$. Однако включение, обратное (1.10), вообще говоря, не верно (см. [2]). 
Лемма 1.5. Пусть $u \in \stackrel{\circ}{H_{2}^{1, q}}(\Omega), \quad \lambda \in[1, q]$. Пусть для любого п-мерного мультииндекса $\alpha, \quad|\alpha|=1$, имеет место включение $\delta^{\alpha} u \in L^{\lambda}(\Omega)$. Тогда $u \in \stackrel{\circ}{W}^{1, \lambda}(\Omega)$.

ДокаЗАТЕЛЬСтво. Ясно, что $\left\{h_{k}(u)\right\} \subset \stackrel{\circ}{W^{1, \lambda}}(\Omega)$. Тогда в силу (1.3), леммы 1.4 и неравенства (1.5) для любого $k \in \mathbb{N}$ имеем

$$
\left(\int_{\Omega}\left|h_{k}(u)\right|^{\lambda^{*}} d x\right)^{1 / \lambda^{*}} \leqslant c_{n, \lambda}\left(\sum_{|\alpha|=1} \int_{\Omega}\left|\delta^{\alpha} u\right|^{\lambda} d x\right)^{1 / \lambda} .
$$

Отсюда, поскольку $h_{k}(u) \rightarrow u$ поточечно в $\Omega$, заключаем, что $u \in L^{\lambda}(\Omega)$. Тогда, учитывая, что $\left|h_{k}(u)\right| \leqslant|u|$ для любого $k \in \mathbb{N}$, имеем $h_{k}(u) \rightarrow u$ сильно в $L^{\lambda}(\Omega)$. Кроме того, в силу лемм $1.3,1.4$ и неравенства (1.5) для любого $n$-мерного мультииндекса $\alpha,|\alpha|=1$, имеем $D^{\alpha} h_{k}(u) \rightarrow \delta^{\alpha} u$ сильно в $L^{\lambda}(\Omega)$. Теперь можно заключить, что $u \in W^{1, \lambda}(\Omega)$ и $h_{k}(u) \rightarrow u$ сильно в $W^{1, \lambda}(\Omega)$. Следовательно, $u \in \stackrel{\circ}{W}^{1, \lambda}(\Omega)$. Лемма доказана.

Лемма 1.6. Пусть $u \in \stackrel{\circ}{H} \underset{2, p}{1, q}(\Omega), \lambda \in[1, p]$ и для любого $n$-мерного мультииндекса $\alpha,|\alpha|=1$, имеет место включение $\delta^{\alpha} u \in L^{2 \lambda}(\Omega)$. Пусть для любого $n$-мерного мультииндекса $\alpha,|\alpha|=2$, имеет место включение $\delta^{\alpha} u \in L^{\lambda}(\Omega)$. Тогда $u \in \stackrel{\circ}{W^{2, \lambda}}(\Omega)$.

ДоКАЗАТЕЛЬСТВо. Ясно, что $\left\{h_{k}(u)\right\} \subset \stackrel{\circ}{W^{2, \lambda}}(\Omega)$. В силу изложенного в доказательстве леммы 1.5 имеем $u \in W^{1, \lambda}(\Omega)$ и $h_{k}(u) \rightarrow u$ сильно в $W^{1, \lambda}(\Omega)$. Кроме того, в силу лемм 1.3 и 1.4 для любого $n$-мерного мультииндекса $\alpha,|\alpha|=2$, имеем $D^{\alpha} h_{k}(u) \rightarrow \delta^{\alpha} u$ сильно в $L^{\lambda}(\Omega)$. Теперь можно заключить, что $u \in W^{2, \lambda}(\Omega)$ и $h_{k}(u) \rightarrow u$ сильно в $W^{2, \lambda}(\Omega)$. Следовательно, $u \in \stackrel{\circ}{W^{2, \lambda}}(\Omega)$. Лемма доказана.

Положим для любых $u \in \stackrel{\circ}{H} \underset{2, p}{1, q}(\Omega)$ и $k \in \mathbb{N}$

$$
I(u, k)=\int_{\Omega}\left\{\sum_{|\alpha|=1}\left|\delta^{\alpha} u\right|^{q}+\sum_{|\alpha|=2}\left|\delta^{\alpha} u\right|^{p}\right\} h_{k}^{\prime}(u) d x .
$$

Лемма 1.7. Пусть $u \in \stackrel{\circ}{H_{2, p}^{1, q}}(\Omega) u k \in \mathbb{N}$. Тогда

$$
\operatorname{meas}\{|u| \geqslant k\} \leqslant c_{n, q}^{q^{*}} k^{-q^{*}}[I(u, k)]^{q^{*} / q} .
$$

ДокАЗАТЕЛЬСТво. Поскольку $\left|h_{k}(u)\right| \geqslant k$ на $\{|u| \geqslant k\}$, имеем

$$
k^{q^{*}} \operatorname{meas}\{|u| \geqslant k\} \leqslant \int_{\Omega}\left|h_{k}(u)\right|^{q^{*}} d x .
$$

Правую часть этого неравенства оценим, используя (1.3), лемму 1.4 и неравенство (1.5). Получим

$$
\int_{\Omega}\left|h_{k}(u)\right|^{q^{*}} d x \leqslant c_{n, q}^{q^{*}}\left(\sum_{|\alpha|=1} \int_{\Omega}\left|D^{\alpha} h_{k}(u)\right|^{q} d x\right)^{q^{*} / q} \leqslant c_{n, q}^{q^{*}}[I(u, k)]^{q^{*} / q} .
$$

Отсюда и из (1.12) выводим (1.11). Лемма доказана. 
ЛЕмма 1.8. Пусть $u \in \stackrel{\circ}{H}_{2, p}^{1, q}(\Omega), \quad \alpha-n$-мерньй мультииндекс, $|\alpha|=1, u$ nycmь $k, k_{1} \in \mathbb{N}$. Тогда

$$
\operatorname{meas}\left\{\left|\delta^{\alpha} u\right| \geqslant k\right\} \leqslant c_{n, q}^{q^{*}} k_{1}^{-q^{*}}\left[I\left(u, k_{1}\right)\right]^{q^{*} / q}+k^{-q} I\left(u, k_{1}\right) .
$$

ДокАЗАТЕЛЬСТво. Положим $G=\left\{|u|<k_{1},\left|\delta^{\alpha} u\right| \geqslant k\right\}$. Имеем

$$
\operatorname{meas}\left\{\left|\delta^{\alpha} u\right| \geqslant k\right\} \leqslant \operatorname{meas}\left\{|u| \geqslant k_{1}\right\}+\operatorname{meas} G,
$$

причем в силу леммы 1.7

$$
\operatorname{meas}\left\{|u| \geqslant k_{1}\right\} \leqslant c_{n, q}^{q^{*}} k_{1}^{-q^{*}}\left[I\left(u, k_{1}\right)\right]^{q^{*} / q}
$$

Оценим meas $G$. Поскольку $k \leqslant\left|\delta^{\alpha} u\right| h_{k_{1}}^{\prime}(u)$ на $G$, имеем

$$
k^{q} \text { meas } G \leqslant \int_{\Omega}\left|\delta^{\alpha} u\right|^{q} h_{k_{1}}^{\prime}(u) d x \leqslant I\left(u, k_{1}\right)
$$

и, следовательно, meas $G \leqslant k^{-q} I\left(u, k_{1}\right)$. Отсюда и из (1.14), (1.15) выводим (1.13). Лемма доказана.

Лемма 1.9. Пусть $u \in \stackrel{\circ}{H_{2}^{1, q}}(\Omega), \quad \alpha-n$-мерный мультииндекс, $|\alpha|=2, u$ nусть $k, k_{1} \in \mathbb{N}$. Тогда

$$
\operatorname{meas}\left\{\left|\delta^{\alpha} u\right| \geqslant k\right\} \leqslant c_{n, q}^{q^{*}} k_{1}^{-q^{*}}\left[I\left(u, k_{1}\right)\right]^{q^{*} / q}+k^{-p} I\left(u, k_{1}\right) .
$$

Доказательство этой леммы аналогично доказательству леммы 1.8.

Положим

$$
r=\frac{n(q-1)}{n-1} .
$$

Поскольку $q \in(2, n)$, имеем $r \in(1, q)$.

ЛЕмма 1.10. Пусть $b_{1}, b_{2}>0,(n-1) / n<\sigma<1, \Phi$ - неотрицательная измеримая функиия на $\Omega$ такая, что $\Phi[\ln (1+\Phi)]^{\sigma} \in L^{1}(\Omega)$. Пусть $u \in \stackrel{\circ}{H}_{2, p}^{1, q}(\Omega)$ и для любого $k \in \mathbb{N}$ справедливо неравенство

$$
I(u, k) \leqslant b_{1} \int_{\Omega} \Phi\left|h_{k}(u)\right| d x+b_{2} .
$$

Тогда:

1) $u \in \stackrel{\circ}{W}^{1, r}(\Omega)$

2) для любого п-мерного мультииндекса $\alpha,|\alpha|=2$, имеем $\delta^{\alpha} u \in L^{r p / q}(\Omega)$;

3) если $r p / q \geqslant 1$, mo $u \in \stackrel{\circ}{W}^{2, r p / q}(\Omega)$. 
ДокАЗАТЕЛЬСТво. Через $b_{i}, i=3,4, \ldots$, будем обозначать положительные постоянные, зависящие только от $n, q, b_{1}, b_{2}, \sigma$, meas $\Omega$ и нормы в $L^{1}(\Omega)$ функции $\Phi[\ln (1+\Phi)]^{\sigma}$.

Положим $q_{1}=\frac{q-1}{2 q}$ и зафиксируем $k \in \mathbb{N}, k>e$. В силу (1.16) имеем

$$
I(u, k) \leqslant b_{1} \int_{\left\{\Phi \leqslant k^{\left.q_{1}\right\}}\right.} \Phi\left|h_{k}(u)\right| d x+b_{1} \int_{\left\{\Phi>k^{q_{1}}\right\}} \Phi\left|h_{k}(u)\right| d x+b_{2} .
$$

Используя неравенство Гёльдера, неравенство (1.3), лемму 1.4 и (1.5), получаем

$$
\begin{aligned}
\int_{\left\{\Phi \leqslant k^{\left.q_{1}\right\}}\right.} \Phi\left|h_{k}(u)\right| d x & \leqslant k^{q_{1}} \int_{\Omega}\left|h_{k}(u)\right| d x \\
& \leqslant k^{q_{1}}(\operatorname{meas} \Omega)^{\left(q^{*}-1\right) / q^{*}}\left(\int_{\Omega}\left|h_{k}(u)\right|^{q^{*}} d x\right)^{1 / q^{*}} \\
& \leqslant k^{q_{1}}(\operatorname{meas} \Omega)^{\left(q^{*}-1\right) / q^{*}} c_{n, q}\left(\sum_{|\alpha|=1} \int_{\Omega}\left|D^{\alpha} h_{k}(u)\right|^{q} d x\right)^{1 / q} \\
& \leqslant b_{3} k^{q_{1}}[I(u, k)]^{1 / q}
\end{aligned}
$$

Учитывая (1.4) и тот факт, что

$$
\Phi<q_{1}^{-\sigma}(\ln k)^{-\sigma} \Phi[\ln (1+\Phi)]^{\sigma} \quad \text { на }\left\{\Phi>k^{q_{1}}\right\}
$$

второй интеграл в правой части (1.17) оценим следующим образом:

$$
\begin{aligned}
\int_{\left\{\Phi>k^{\left.q_{1}\right\}}\right.} & \Phi\left|h_{k}(u)\right| d x \leqslant 2 k \int_{\left\{\Phi>k^{\left.q_{1}\right\}}\right.} \Phi d x \\
& \leqslant 2 k q_{1}^{-\sigma}(\ln k)^{-\sigma} \int_{\Omega} \Phi[\ln (1+\Phi)]^{\sigma} d x=b_{4} k(\ln k)^{-\sigma} .
\end{aligned}
$$

Из соотношений (1.17)-(1.19), используя неравенство Юнга, получаем

$$
\begin{aligned}
I(u, k) & \leqslant b_{1} b_{3} k^{q_{1}}[I(u, k)]^{1 / q}+b_{1} b_{4} k(\ln k)^{-\sigma}+b_{2} \\
& \leqslant \frac{1}{q} I(u, k)+\frac{q-1}{q} b_{5}\left[k^{1 / 2}+k(\ln k)^{-\sigma}\right] .
\end{aligned}
$$

Отсюда заключаем, что для любого $k \in \mathbb{N}, k>e$, справедливо неравенство

$$
I(u, k) \leqslant b_{6} k(\ln k)^{-\sigma} .
$$

Далее, пусть $\alpha$ - произвольный $n$-мерный мультииндекс такой, что $|\alpha|=1$. Зафиксируем $k \in \mathbb{N}, k>e^{(n-1) /(n-q)}$. Ясно, что сушествует $\eta>e$ такое, что

$$
\eta^{n-1}(\ln \eta)^{\sigma}=k^{n-q}
$$


Из этого равенства следует, что

$$
\begin{gathered}
\eta^{-r^{*}}(\ln \eta)^{-\sigma n /(n-q)}=k^{-q} \eta(\ln \eta)^{-\sigma}=k^{-r}(\ln \eta)^{-\sigma n /(n-1)} \\
k^{n-q} \leqslant \eta^{n} .
\end{gathered}
$$

Пусть $k_{1}$ - минимальное натуральное число, большее $\eta$. Из соотношений (1.21) и (1.22) вытекают неравенства

$$
\begin{aligned}
k_{1}^{-r^{*}}\left(\ln k_{1}\right)^{-\sigma n /(n-q)} & \leqslant b_{7} k^{-r}(\ln k)^{-\sigma n /(n-1)}, \\
k^{-q} k_{1}\left(\ln k_{1}\right)^{-\sigma} & \leqslant b_{8} k^{-r}(\ln k)^{-\sigma n /(n-1)} .
\end{aligned}
$$

Кроме того, поскольку $k_{1}>e$, в силу (1.20) имеем

$$
I\left(u, k_{1}\right) \leqslant b_{6} k_{1}\left(\ln k_{1}\right)^{-\sigma} .
$$

Теперь, используя лемму 1.8 и неравенства (1.23)-(1.25), получаем

$$
\text { meas }\left\{\left|\delta^{\alpha} u\right| \geqslant k\right\} \leqslant b_{9} k^{-r}(\ln k)^{-\sigma n /(n-1)} .
$$

Тогда в силу неравенства $\sigma>(n-1) / n$ и леммы 1.2 имеем $\delta^{\alpha} u \in L^{r}(\Omega)$.

Таким образом, для любого $n$-мерного мультииндекса $\alpha,|\alpha|=1$, имеем $\delta^{\alpha} u \in$ $L^{r}(\Omega)$. Отсюда и из леммы 1.5 выводим, что $u \in \stackrel{\circ}{W}{ }^{1, r}(\Omega)$, и тем самым утверждение 1) леммы доказано.

Перейдем к доказательству утверждения 2). Пусть $\alpha$-произвольный $n$-мерный мультииндекс такой, что $|\alpha|=2$. Зафиксируем $k \in \mathbb{N}, k>e^{q(n-1) / p(n-q)}$. Ясно, что существует $y>e$ такое, что

$$
y^{n-1}(\ln y)^{\sigma}=k^{p(n-q) / q} .
$$

Из этого равенства следует, что

$$
\begin{aligned}
y^{-r^{*}}(\ln y)^{-\sigma n /(n-q)}= & k^{-p} y(\ln y)^{-\sigma}=k^{-r p / q}(\ln y)^{-\sigma n /(n-1)}, \\
& k^{p(n-q)} \leqslant y^{q n} .
\end{aligned}
$$

Пусть $k_{2}$ - минимальное натуральное число, большее $y$. Из соотношений (1.26) и (1.27) вытекают неравенства

$$
\begin{aligned}
k_{2}^{-r^{*}}\left(\ln k_{2}\right)^{-\sigma n /(n-q)} & \leqslant b_{10} k^{-r p / q}(\ln k)^{-\sigma n /(n-1)}, \\
k^{-p} k_{2}\left(\ln k_{2}\right)^{-\sigma} & \leqslant b_{11} k^{-r p / q}(\ln k)^{-\sigma n /(n-1)} .
\end{aligned}
$$

Кроме того, поскольку $k_{2}>e$, в силу (1.20) имеем

$$
I\left(u, k_{2}\right) \leqslant b_{6} k_{2}\left(\ln k_{2}\right)^{-\sigma} \text {. }
$$


Используя лемму 1.9 и неравенства (1.28)-(1.30), устанавливаем, что

$$
\operatorname{meas}\left\{\left|\delta^{\alpha} u\right| \geqslant k\right\} \leqslant b_{12} k^{-r p / q}(\ln k)^{-\sigma n /(n-1)} .
$$

Тогда в силу неравенства $\sigma>(n-1) / n$ и леммы 1.2 имеем $\delta^{\alpha} u \in L^{r p / q}(\Omega)$. Тем самым справедливость утверждения 2) леммы доказана.

Остается установить справедливость утверждения 3) леммы. Предположим, что $r p / q \geqslant 1$. Тогда $r p / q \in[1, p]$. Поскольку $2 p<q$, имеем $2 r p / q<r$, и тогда в силу установленного вьше, при доказательстве утверждения 1) леммы, для любого $n$-мерного мультииндекса $\alpha,|\alpha|=1$, имеем $\delta^{\alpha} u \in L^{2 r p / q}(\Omega)$. Теперь, учитывая утверждение 2$)$ и используя лемму 1.6 , получаем, что $u \in \stackrel{\circ}{W^{2}}, r p / q(\Omega)$. Таким образом, утверждение 3) леммы справедливо, и тем самьм ее доказательство завершено.

Лемма 1.11. Пусть $b^{\prime}, b^{\prime \prime}>0,1 \leqslant m<n q /(n q-n+q), \Phi \in L^{m}(\Omega), \Phi \geqslant 0$ на $\Omega$. Пусть $и \in \stackrel{\circ}{H_{2, p}^{1, q}}(\Omega)$ и для любого $k \in \mathbb{N}$ справедливо неравенство

$$
I(u, k) \leqslant b^{\prime} \int_{\Omega} \Phi\left|h_{k}(u)\right| d x+b^{\prime \prime} .
$$

Тогда:

1) если $\lambda \in\left[1,(q-1) m^{*}\right)$, mo $u \in \stackrel{\circ}{W}^{1, \lambda}(\Omega)$;

2) если $\alpha-n$-мерный мультииндекс, $|\alpha|=2$, и $\lambda \in\left(0, \frac{p}{q}(q-1) m^{*}\right)$, то $\delta^{\alpha} u \in L^{\lambda}(\Omega)$

3) ecлu $\frac{p}{q}(q-1) m^{*}>1 u \lambda \in\left[1, \frac{p}{q}(q-1) m^{*}\right)$, mo $u \in \stackrel{\circ}{W^{2, \lambda}}(\Omega)$.

ДокАЗАТЕЛЬСТво. Прежде всего заметим, что в силу условия леммы имеем $m q<n,(m-1) q^{*}<m$.

Положим

$$
m_{1}=1-\frac{m-1}{m} q^{*}, \quad m_{2}=\frac{(m-1) q^{*}}{m q}, \quad m_{3}=\frac{n-m q}{n-m}, \quad \theta=\frac{n m(q-1)}{n-m q} .
$$

Ясно, что $0<m_{1} \leqslant 1,0 \leqslant m_{2}<1$. Кроме того, имеем

$$
\begin{gathered}
q^{*}\left[1-\frac{m_{1}}{\left(1-m_{2}\right) q}\right]=\theta \\
m_{3} \theta=q-\frac{m_{1} m_{3}}{1-m_{2}}
\end{gathered}
$$

Далее через $b_{i}, i=13,14, \ldots$, будем обозначать положительные постоянные, зависяшие только от $n, q, b^{\prime}, b^{\prime \prime}, m$ и нормы в $L^{m}(\Omega)$ функции $\Phi$.

Зафиксируем произвольное $k \in \mathbb{N}$. Предположим, что $m>1$. Тогда $m_{1}<1$ и, используя $(1.31),(1.4)$ и неравенство Гёльдера, получаем

$$
\begin{aligned}
I(u, k) & \leqslant b^{\prime}(2 k)^{m_{1}} \int_{\Omega} \Phi\left|h_{k}(u)\right|^{1-m_{1}} d x+b^{\prime \prime} \\
& \leqslant b^{\prime}(2 k)^{m_{1}}\|\Phi\|_{L^{m}(\Omega)}\left(\int_{\Omega}\left|h_{k}(u)\right|^{q^{*}} d x\right)^{(m-1) / m}+b^{\prime \prime} .
\end{aligned}
$$


Отсюда и из соотношений $(1.3),(1.5)$ и леммы 1.4 вытекает, что

$$
I(u, k) \leqslant b_{13} k^{m_{1}}[I(u, k)]^{m_{2}}+b^{\prime \prime} .
$$

Следовательно,

$$
I(u, k) \leqslant b_{14} k^{m_{1} /\left(1-m_{2}\right)} .
$$

Легко проверить, что это же неравенство верно и в случае $m=1$.

Таким образом, доказано, что для любого $k \in \mathbb{N}$ имеет место неравенство (1.34).

Далее, пусть $\lambda \in\left[1,(q-1) m^{*}\right)$ и $\alpha-n$-мерньй мультииндекс такой, что $|\alpha|=1$. Зафиксируем $k \in \mathbb{N}$, а также $k_{1} \in \mathbb{N}$ такое, что

$$
k^{m_{3}}<k_{1} \leqslant 2 k^{m_{3}}
$$

В силу (1.34) и (1.35) имеем $I\left(u, k_{1}\right) \leqslant b_{15} k^{m_{1} m_{3} /\left(1-m_{2}\right)}$. Используя это неравенство, лемму 1.8 и соотношения (1.35), (1.32), (1.33), находим

$$
\operatorname{meas}\left\{\left|\delta^{\alpha} u\right| \geqslant k\right\} \leqslant b_{16} k^{-m_{3} \theta} \text {. }
$$

Тогда в силу леммы $1.1 \delta^{\alpha} u \in L^{\lambda}(\Omega)$. Отсюда, учитьвая неравенство $(q-1) m^{*}<q$ и применяя лемму 1.5 , выводим, что $u \in \stackrel{\circ}{W^{1}}, \lambda(\Omega)$. Тем самым утверждение 1$)$ леммы доказано.

Перейдем к доказательству утверждения 2$)$ леммы. Пусть $\alpha-n$-мерный мультииндекс, $|\alpha|=2$, и $\lambda \in\left(0, \frac{p}{q}(q-1) m^{*}\right)$. Зафиксируем $k \in \mathbb{N}$, а также $k_{2} \in \mathbb{N}$ такое, что

$$
k^{p m_{3} / q}<k_{2} \leqslant 2 k^{p m_{3} / q} .
$$

В силу $(1.34)$ и $(1.36)$ имеем $I\left(u, k_{2}\right) \leqslant b_{17} k^{p m_{1} m_{3} / q\left(1-m_{2}\right)}$. Используя это неравенство, лемму 1.9 и соотношения (1.36), (1.32), (1.33), получаем

$$
\operatorname{meas}\left\{\left|\delta^{\alpha} u\right| \geqslant k\right\} \leqslant b_{18} k^{-p m_{3} \theta / q}
$$

Теперь, учитьвая лемму 1.1 , заключаем, что $\delta^{\alpha} u \in L^{\lambda}(\Omega)$, и тем самым утверждение 2) леммы доказано.

Докажем утверждение 3$)$. Пусть $\frac{p}{q}(q-1) m^{*}>1$ и $\lambda \in\left[1, \frac{p}{q}(q-1) m^{*}\right)$. Имеем $u \in \stackrel{\circ}{H}_{2, p}^{1, q}(\Omega), \lambda \in[1, p]$. Кроме того, $2 \lambda \in\left[1,(q-1) m^{*}\right)$, и тогда в силу установленного при доказательстве утверждения 1 ) для любого $n$-мерного мультииндекса $\alpha,|\alpha|=1$, имеем $\delta^{\alpha} u \in L^{2 \lambda}(\Omega)$. Наконец, согласно утверждению 2$)$ для любого $n$-мерного мультииндекса $\alpha,|\alpha|=2$, имеем $\delta^{\alpha} u \in L^{\lambda}(\Omega)$. Используя теперь лемму 1.6 , заключаем, что $u \in \stackrel{\circ}{W^{2}}, \lambda(\Omega)$. Таким образом, утверждение 3 ) леммы справедливо, и тем самым ее доказательство завершено. 


\section{$\S 2$. О суммируемости решений задачи Дирихле}

Пусть $c_{1}, c_{2}>0, g_{1}, g_{2}$-неотрицательные функции на $\Omega, g_{1}, g_{2} \in L^{1}(\Omega)$, и пусть $A_{\alpha}: \Omega \times \mathbb{R}^{n, 2} \rightarrow \mathbb{R}$ - функция Каратеодори для любого $\alpha \in \Lambda$. Будем предполагать, что для почти всех $x \in \Omega$ и любых $\xi \in \mathbb{R}^{n, 2}$ справедливы неравенства

$$
\begin{gathered}
\sum_{|\alpha|=1}\left|A_{\alpha}(x, \xi)\right|^{q /(q-1)}+\sum_{|\alpha|=2}\left|A_{\alpha}(x, \xi)\right|^{p /(p-1)} \\
\leqslant c_{1}\left\{\sum_{|\alpha|=1}\left|\xi_{\alpha}\right|^{q}+\sum_{|\alpha|=2}\left|\xi_{\alpha}\right|^{p}\right\}+g_{1}(x), \\
\sum_{\alpha \in \Lambda} A_{\alpha}(x, \xi) \xi_{\alpha} \geqslant c_{2}\left\{\sum_{|\alpha|=1}\left|\xi_{\alpha}\right|^{q}+\sum_{|\alpha|=2}\left|\xi_{\alpha}\right|^{p}\right\}-g_{2}(x) .
\end{gathered}
$$

Пусть $f \in L^{1}(\Omega)$. Рассмотрим следуюшую задачу Дирихле:

$$
\begin{array}{ll}
\sum_{\alpha \in \Lambda}(-1)^{|\alpha|} D^{\alpha} A_{\alpha}\left(x, \nabla_{2} u\right)=f & \text { в } \Omega, \\
D^{\alpha} u=0, \quad|\alpha|=0,1, & \text { на } \partial \Omega .
\end{array}
$$

Введем отображение: если $u \in \stackrel{\circ}{H}_{2, p}^{1, q}(\Omega)$, то $\delta_{2} u: \Omega \rightarrow \mathbb{R}^{n, 2}$, причем для любых $x \in \Omega$ и $\alpha \in \Lambda$ имеем $\left(\delta_{2} u(x)\right)_{\alpha}=\delta^{\alpha} u(x)$.

Заметим, что если $u \in \stackrel{\circ}{H_{2, p}^{1, q}}(\Omega), \varphi \in C_{0}^{\infty}(\Omega), k \in \mathbb{N}$ и $\alpha \in \Lambda$, то сушествуют конечные интегралы функций $A_{\alpha}\left(x, \delta_{2} u\right) \delta^{\alpha} u$ и $A_{\alpha}\left(x, \delta_{2} u\right) \delta^{\alpha} \varphi$ по множеству $\{|u-\varphi|<2 k\}$. Это вытекает из (2.1) и леммы 1.3 .

ОПРЕДЕЛЕНИЕ 2.1. Энтропийным решением задачи (2.3), (2.4) будем называть функцию $u \in \stackrel{\circ}{H} \underset{2, p}{1, q}(\Omega)$, удовлетворяющую условию: существуют $c>0$, $b \in(1, r)$ и $\gamma>0$ такие, что для любых $\varphi \in C_{0}^{\infty}(\Omega)$ и $k \in \mathbb{N}[1],[2]$

$$
\begin{array}{r}
\int_{\{|u-\varphi|<2 k\}}\left\{\sum_{\alpha \in \Lambda} A_{\alpha}\left(x, \delta_{2} u\right)\left(\delta^{\alpha} u-\delta^{\alpha} \varphi\right)\right\} h_{k}^{\prime}(u-\varphi) d x \\
\leqslant \int_{\Omega} f h_{k}(u-\varphi) d x+c\left[1+\|\varphi\|_{W^{1, b}(\Omega)}\right]^{b} k^{-\gamma} .
\end{array}
$$

Положим

$$
p_{1}=\frac{(3 n-2) p}{n+p-1}, \quad p_{2}=\frac{n p}{n(p-1)+1} .
$$

Поскольку $p \in(1, n / 2)$, имеем $p_{1} \in(2 p, n), p_{2} \in(1, n)$.

ЗАмЕчАнИЕ 2.1. Из результатов [1], [2] следует, что если $q>p_{1}$ и для почти всех $x \in \Omega$ и любых $\xi, \xi^{\prime} \in \mathbb{R}^{n, 2}, \xi \neq \xi^{\prime}$, имеет место неравенство

$$
\sum_{\alpha \in \Lambda}\left[A_{\alpha}(x, \xi)-A_{\alpha}\left(x, \xi^{\prime}\right)\right]\left(\xi_{\alpha}-\xi_{\alpha}^{\prime}\right)>0,
$$

то сушествует единственное энтропийное решение $u$ задачи $(2.3),(2.4)$, причем

1) для любого $\alpha \in \Lambda A_{\alpha}\left(x, \delta_{2} u\right) \in L^{1}(\Omega)$;

2) для любой функции $\varphi \in C_{0}^{\infty}(\Omega)$

$$
\int_{\Omega}\left\{\sum_{\alpha \in \Lambda} A_{\alpha}\left(x, \delta_{2} u\right) \delta^{\alpha} \varphi\right\} d x=\int_{\Omega} f \varphi d x .
$$


ЛЕмма 2.1. Пусть и - әнтропийное решение задачи (2.3), (2.4). Тогда существуют $c^{\prime}, c^{\prime \prime}>0$ такие, что для любого $k \in \mathbb{N}$

$$
I(u, k) \leqslant c^{\prime} \int_{\Omega}|f|\left|h_{k}(u)\right| d x+c^{\prime \prime}
$$

ДокАЗАТЕЛЬСтво. В силу определения 2.1 сушествует $c>0$ такое, что для любого $k \in \mathbb{N}$

$$
\int_{\{|u|<2 k\}}\left\{\sum_{\alpha \in \Lambda} A_{\alpha}\left(x, \delta_{2} u\right) \delta^{\alpha} u\right\} h_{k}^{\prime}(u) d x \leqslant \int_{\Omega} f h_{k}(u) d x+c .
$$

Положим

$$
c^{\prime}=\frac{1}{c_{2}}, \quad c^{\prime \prime}=\frac{1}{c_{2}}\left(\left\|g_{2}\right\|_{L^{1}(\Omega)}+c\right) .
$$

Зафиксируем $k \in \mathbb{N}$. Используя (2.2) и (1.5), устанавливаем, что

$$
c_{2} I(u, k) \leqslant \int_{\{|u|<2 k\}}\left\{\sum_{\alpha \in \Lambda} A_{\alpha}\left(x, \delta_{2} u\right) \delta^{\alpha} u\right\} h_{k}^{\prime}(u) d x+\left\|g_{2}\right\|_{L^{1}(\Omega)}
$$

Отсюда и из неравенства (2.7) выводим (2.6). Лемма доказана.

TЕOPEMA 2.1. Пусть $(n-1) / n<\sigma<1$ uf $[\ln (1+|f|)]^{\sigma} \in L^{1}(\Omega)$. Пусть $u-$ энтропийное решение задачи (2.3), (2.4). Тогда:

1) $u \in \stackrel{\circ}{W}^{1, r}(\Omega)$;

2) для любого п-мерного мультииндекса $\alpha,|\alpha|=2$, имеем $\delta^{\alpha} u \in L^{r p / q}(\Omega)$;

3) если $q \geqslant p_{2}$, то $u \in \stackrel{\circ}{W}^{2, r p / q}(\Omega)$.

Этот результат вытекает из лемм 2.1 и 1.10.

СЛЕДСТВИЕ 2.1. Пусть $p \geqslant 3 / 2-1 / n$, и пусть выполняются условия теореми 2.1. Тогда $u \in \stackrel{\circ}{W^{1, r}}(\Omega) \cap \stackrel{\circ}{W^{2, r p} / q}(\Omega)$.

Для доказательства этого результата достаточно воспользоваться теоремой 2.1, а затем учесть, что $q>2 p$ и в силу неравенства $p \geqslant 3 / 2-1 / n$ справедливо неравенство $p_{2} \leqslant 2 p$.

TеOрема 2.2. Пусть $1 \leqslant m<n q /(n q-n+q) u f \in L^{m}(\Omega)$. Пусть $u-$ энтропийное решение задачи (2.3), (2.4). Тогда:

1) если $\lambda \in\left[1,(q-1) m^{*}\right)$, то $u \in \stackrel{\circ}{W^{1, \lambda}}(\Omega)$;

2) если $\alpha-n$-мерный мультииндекс, $|\alpha|=2, u \lambda \in\left(0, \frac{p}{q}(q-1) m^{*}\right)$, mо $\delta^{\alpha} u \in L^{\lambda}(\Omega)$;

3) если $\frac{p}{q}(q-1) m^{*}>1 u \lambda \in\left[1, \frac{p}{q}(q-1) m^{*}\right)$, mo $u \in \stackrel{\circ}{W}^{2, \lambda}(\Omega)$.

Этот результат вытекает из лемм 2.1 и 1.11. 
СлЕДСТВИЕ 2.2. Пусть $q>p_{2}$, и пусть выполняются условия теоремы 2.2. Тогда $\frac{p}{q}(q-1) m^{*}>1$, и для любого $\lambda \in\left[1, \frac{p}{q}(q-1) m^{*}\right)$ имеем $u \in \stackrel{\circ}{W^{1,2 \lambda}}(\Omega) \cap$ $\stackrel{\circ}{W}^{2, \lambda}(\Omega)$.

Для доказательства этого следствия достаточно воспользоваться теоремой 2.2 и тем, что из $q>p_{2}$ вытекает неравенство $\frac{p}{q}(q-1) m^{*}>1$.

ЗАМЕчАНИЕ 2.2 . Нетрудно проверить, что если $q \leqslant p_{2}$, то $n q /(n p q-n p+q) \geqslant 1$.

СлЕДСТВИЕ 2.3. Пусть $q \leqslant p_{2}, \quad n q /(n p q-n p+q)<m<n q /(n q-n+q)$ u $f \in L^{m}(\Omega)$. Пусть и - энтропийное решение задачи (2.3), (2.4). Тогда $\frac{p}{q}(q-1) m^{*}>1$ и для любого $\lambda \in\left[1, \frac{p}{q}(q-1) m^{*}\right)$ имеем $u \in \stackrel{\circ}{W^{1,2 \lambda}}(\Omega) \cap \stackrel{\circ}{W^{2, \lambda}}(\Omega)$.

Этот результат вытекает из теоремы 2.2 и замечания 2.2 .

В заключение приведем несколько утверждений для слабых решений задачи (2.3), (2.4).

ОПРЕДЕЛЕНИЕ 2.2. $W$-решением задачи (2.3), (2.4) будем называть функцию $u \in \stackrel{\circ}{W}^{2,1}(\Omega)$, удовлетворяющую условиям:

1) для любого $\alpha \in \Lambda A_{\alpha}\left(x, \nabla_{2} u\right) \in L^{1}(\Omega)$;

2) для любой функции $\varphi \in C_{0}^{\infty}(\Omega)$

$$
\int_{\Omega}\left\{\sum_{\alpha \in \Lambda} A_{\alpha}\left(x, \nabla_{2} u\right) D^{\alpha} \varphi\right\} d x=\int_{\Omega} f \varphi d x
$$

ЗАмечание 2.3 . Из результатов работы $[2, \S 7$, леммы $2.5,2.6,8.1,8.2]$ и лемм $1.4,1.6$ настоящей статьи вытекает, что если $q>p_{2}$ и для почти всех $x \in \Omega$ и любых $\xi, \xi^{\prime} \in \mathbb{R}^{n, 2}, \xi \neq \xi^{\prime}$, имеет место неравенство (2.5), то сушествуют $W$-решение $u$ задачи $(2.3),(2.4)$ и положительные постоянные $c^{\prime}, c^{\prime \prime}$ такие, что $u \in \stackrel{\circ}{H_{2, p}^{1, q}}(\Omega)$ и для любого $k \in \mathbb{N}$ справедливо неравенство (2.6).

TЕОРема 2.3. Пусть $q>p_{2}$ и для почти всех $x \in \Omega$ и любьх $\xi, \xi^{\prime} \in \mathbb{R}^{n, 2}$, $\xi \neq \xi^{\prime}$, имеет место неравенство (2.5). Пусть $(n-1) / n<\sigma<1$ и $f[\ln (1+$ $|f|)]^{\sigma} \in L^{1}(\Omega)$. Тогда существует $W$-решение и задачи (2.3), (2.4) такое, что $u \in \stackrel{\circ}{W}^{1, r}(\Omega) \cap \stackrel{\circ}{W}^{2, r p / q}(\Omega)$.

TEOPEMA 2.4. Пусть $q>p_{2}$ и для почти всех $x \in \Omega$ u любы $\xi, \xi^{\prime} \in \mathbb{R}^{n, 2}$, $\xi \neq \xi^{\prime}$, имеет место неравенство (2.5). Пусть $1 \leqslant m<n q /(n q-n+q) u$ $f \in L^{m}(\Omega)$. Тогда существует $W$-решение и задачи (2.3), (2.4) такое, что для любого $\lambda \in\left[1, \frac{p}{q}(q-1) m^{*}\right)$ справедливо включение $u \in \stackrel{\circ}{W^{1,2 \lambda}}(\Omega) \cap \stackrel{\circ}{W^{2, \lambda}}(\Omega)$.

Сформулированные теоремы являются следствиями замечания 2.3 и лемм 1.10 , 1.11.

ЗАМЕчАнИЕ 2.4. Если $m \geqslant n q /(n q-n+q), f \in L^{m}(\Omega)$ и для почти всех $x \in \Omega$ и любых $\xi, \xi^{\prime} \in \mathbb{R}^{n, 2}$ имеет место неравенство

$$
\sum_{\alpha \in \Lambda}\left[A_{\alpha}(x, \xi)-A_{\alpha}\left(x, \xi^{\prime}\right)\right]\left(\xi_{\alpha}-\xi_{\alpha}^{\prime}\right) \geqslant 0,
$$


то сушествует $W$-решение $u$ задачи $(2.3),(2.4)$ такое, что $u \in \stackrel{\circ}{W} \underset{2, p}{1, q}(\Omega)$.

Это следует из того, что при указанных условиях функция $f$ естественным образом порождает линейный непрерывный функционал на $\stackrel{\circ}{W_{2, p}^{1, q}}(\Omega)$ и рассматриваемая задача вкладывается в обычную схему теории монотонных операторов (см., например, [8]).

\section{Список литературы}

1. Kovalevsky A. Entropy solutions of Dirichlet problem for a class of nonlinear elliptic fourth order equations with $L^{1}$-data // Nonlinear Boundary Value Problems. 1999. V. 9. P. $46-54$.

2. Ковалевский A.A. Энтропийные решения задачи Дирихле для одного класса нелинейных эллиптических уравнений четвертого порядка с $L^{1}$-правыми частями // Изв. РАН. Сер. матем. 2001. Т. 65. № 2. С. 27-80.

3. Bénilan Ph., Boccardo L., Gallouët T., Gariepy R., Pierre M., Vazquez J.L. An $L^{1}$-theory of existence and uniqueness of solutions of nonlinear elliptic equations // Ann. Scuola Norm. Sup. Pisa Cl. Sci. 1995. V. 22. P. 241-273.

4. Boccardo L., Gallouët T. Non-linear elliptic and parabolic equations involving measure data // J. Funct. Anal. 1989. V. 87. P. 149-169.

5. Boccardo L., Gallouët T. Nonlinear elliptic equations with right hand side measures // Comm. Partial Differential Equations. 1992. V. 17. P. 641-655.

6. Ковалевский $A$. A. О суммируемости решений нелинейных эллиптических уравнений с правыми частями из классов, близких к $L^{1} / /$ Матем. заметки. 2001. Т. 70 . №3. C. $375-385$.

7. Gilbarg D., Trudinger N.S. Elliptic partial differential equations of second order. Berlin: Springer-Verlag, 1983.

8. Lions J. L. Quelques méthodes de résolution des problèmes aux limites non linéaires. Paris: Dunod, Gauthier-Villars, 1969.

E-mail: alexkvl@iamm.ac.donetsk.ua

Поступило в редакцию

31.I.2001 